\title{
A Solid-State NMR Study of Water in Poly(vinyl butyral) by Magic Angle Spinning
}

\author{
Soon Yong Jeong ${ }^{+*}$ and Oc Hee Han ${ }^{\ddagger ॰}$ \\ †Department of Chemistry, Kungpook National Chiversin, Daegu 702-701. Korea \\ ¥. Analvisis Research Division, Daegu Center, Korea Basic. Science Institute. Daegu 702-701, Korea \\ "E-mail: ohhaniakbsire.kr \\ Received January 12.2007
}

\begin{abstract}
Poly(vinyl butyral) (PVB) with different wt $\%$ water was studied gravimetrically as well as with ${ }^{1} \mathrm{H}$ magic angle spinning (MAS) nuclear magnetic resonance (NMR). The composition of PVB samples changes during MAS NMR because of the centrifugal force. As MAS time progresses, initially free water was removed fast but bound water also was gradually depleted. More water was diminished at faster spinuing speeds. longer spinning time. higher temperatures. and higher initial water contents. As water in PVB was reduced. the chemical shifts and line widths of different types of water and also those of PVB changed. Our results demonstrate that ${ }^{1} \mathrm{H}$ MAS NMR carried out at $10 \mathrm{kHz}$ in less than about 5 minutes is a convenient and sensitive technique to measure: (a) the content variations of different types of water in polymers, (b) the degree of the interaction of water and polymer. and (c) the molecular dynamics of the polymer. Our study can be extended to different soft polymers with other small molecules than water in them.
\end{abstract}

Key Words : Sample spinning. Hydrated polymer. ${ }^{1} \mathrm{H}$ NMR. Poly(vinyl butyral)

\section{Introduction}

Water can play an important role in determining the properties of the polymers such as mechanical tensile and compressional strengths, elastic elongation. and plasticity. ${ }^{1}$ Water plays a key role also in the biological function of proteins such as enzyme catalysis, folding, architecture. dynamics and conformational stability. ${ }^{2}$ Hence the interaction between water and polymers or biopolymers has been studied extensively. ${ }^{1.6}$ however, the details of the molecular level interactions between water and polymer is not fully unfolded yet. One of the representative spectroscopic techniques to probe them is magic angle spinning (MAS). a routinely used high resolution solid-state nuclear magnetic resonance (NMR) teclnnique to study molecular structures and dynamics of polymer and biopolymer. But it was reported that by centrifugal force of MAS water can be removed from samples ${ }^{7}$ or redistributed in the sample container. ${ }^{8}$ In addition, phase separation of water can occur and water relaxation times can vary in the samples. ${ }^{9}$

Poly(vinyl butyral) (PVB) has been used as a main ingredient of a plastic interlayer in laminated glass. ${ }^{10}$ PVB is typically synthesized from butylaldehyde and poly(vinyl alcohol) resulting in a copolymer composed of butyral ring structures and hydrophilic vinyl alcohol units. This procedure often results in inhomogeneous acetylation distribution over the product ${ }^{16}$ and irreproducible mechanical properties after drying the collected PVB from water. To reduce this irreproducibility. first it is necessary to understand the interaction between water and PVB at the molecular level. especially during water removal process. In this work, PVB samples with different initial water contents were studied gravinetrically and by ${ }^{1} \mathrm{H}$ MAS experiments at different spiuning rates and temperatures to monitor the water con- tents in the PVB samples and the molecular dynamics variation of PVB resulting from the water reduction. Instead of ${ }^{13} \mathrm{C}$ NMR frequently used for polymer studies, ${ }^{1} \mathrm{H}$ NMR was chosen to observe water directly and to have a short signal acquisition time.

\section{Experimental}

PVB samples with 9 or 18 weight percent water content. denoted $9 \%$ PVB or $18 \%$ PVB, respectively. were supplied from the Polymer Research Laboratory. Research Center. Oriental Chemical Industries in Korea. Our samples were prepared by precipitating PVB into water after acetylation.

All NMR experiments were carried out with a rotor of 4 min diameter and on a DSX $400 \mathrm{MHz}$ NMR spectrometer system (Bruker Biospin Corporation, Germany) with a 9.4 Tesla wide-bore magnet. Sample spining rates employed were 4.5 and $10 \mathrm{kHz}$ and spin rate stability was within \pm 4 $\mathrm{Hz}$. Five or six ${ }^{1} \mathrm{H}$ MAS spectra were collected during a 24 hour period. During the spectral acquisition. the same spinning rate of $10 \mathrm{kHz}$. which was confirmed not to influence the samples for a measuring period of less than 5 minutes, was employed for all the samples. A pulse length of $2 \mu \mathrm{s}$, a repetition delay of $3 \mathrm{~s}, 16$ scans, and a spectral width of 100 $\mathrm{kHz}$ were used for obtaining a ${ }^{\mathrm{j}} \mathrm{H}$ MAS spectrum. For variable temperature experiments, the sample was continuously spun at $10 \mathrm{kHz}$ and the spectrum was recorded after the sample was equilibrated at a given temperature. Here the temperature and spinuing duration are independent variables.

The total weight of a rotor and PVB sample in it was measured 6 or 7 times during the 24 hours experiment to record weight variation.

${ }^{1} \mathrm{H}$ NMR peak areas were converted to the number of protons observed using the proton quantity per unit peak 


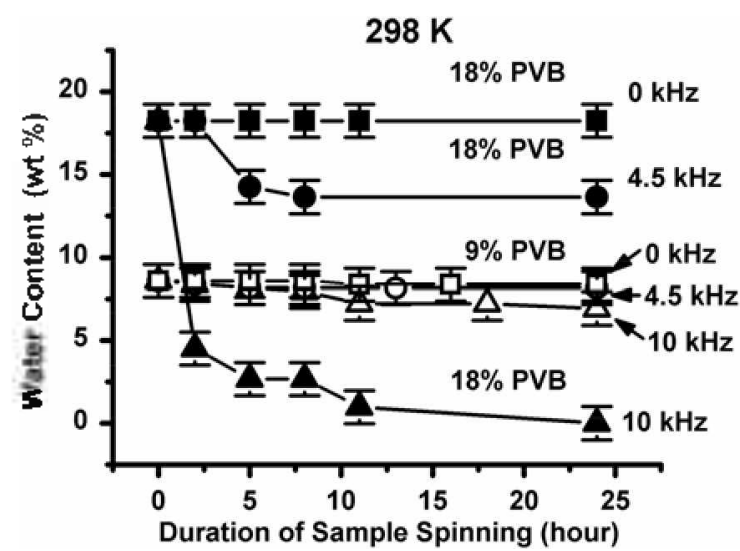

Figure 1. Plot of water contents of $18 \%$ PVB (filled shapes) and $9 \%$ PVB (unfilled shapes) versus duration of sample spinuing at $298 \mathrm{~K}$. The samples spun at 4.5 (circle) and $10 \mathrm{kHz}$ (triangle) spinning rates were compared with the sample not spun (square)

area which was calculated with respect to the total ${ }^{~} \mathrm{H}$ peak area reduced and the weight loss of $18 \%$ PVB after MAS experiment at $10 \mathrm{kHz}$ and room temperature for 24 hours. on the basis that the weight reduction is from the water loss

\section{Results and Discussion}

The weight loss of the $9 \%$ and 18\% PVB samples was detected as shown in Figure 1 as MAS time elapses. The weight loss was faster for higher spinning and initial water content while those samples not spun did not lose water. However, the weight loss of the $9 \%$ PVB sample was much less than that of the $18 \%$ PVB sample in a given spinuing rate and duration. This is explained by that more waters in

(a)

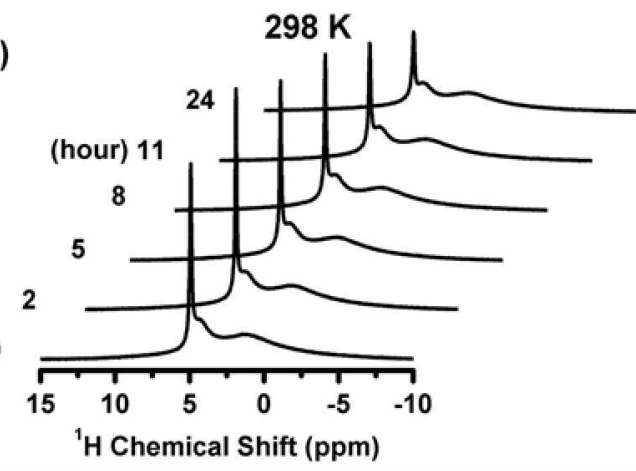

(b)

$298 \mathrm{~K}$

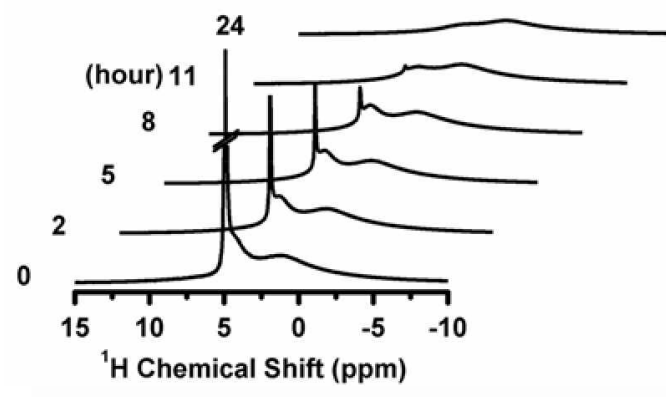

$9 \%$ PVB are tightly bound to PVB than in $18 \%$ PVB. The possibility to lose species other than water during spinning was checked with gas chromatography-mass spectrometer experiments but no noticeable organic species was detected.

To study the weight loss mechanism in further detail. ${ }^{1} \mathrm{H}$ MAS NMR was employed. No spectral changes in ${ }^{1} \mathrm{H}$ MAS spectra were observed for the PVB samples kept in a rotor closed but not spun for 24 hours. In contrast. as the sample was continuously spun at 4.5 or $10 \mathrm{kHz}$, the sharp peak near $4.9 \mathrm{ppm}$ diminished [Figures 2(a) and 2(b)]. The falling off of the signal was faster for higher spinning rate and initial water content. overall consistent with the gravimetric data. For quantitative information, the 'H MAS NMR spectra were deconvoluted as representatively shown in Figure 3. The peaks were assigned according to reference 11 and summarized in Table 1. However, for the signal not resolved due to restricted mobility the spectral deconvolution into individual peaks of distinct proton species is problematic since the strong proton homonuclear dipole interaction makes the proton species lose their identities. In our spectra. methyl. metlylene. and methine peaks were not well resolved while the peaks of free water or lydroxyl and bound water stand out with narrow line widths. Therefore. the deconvoluted peaks were grouped and the resulting three groups of the peaks were assigned for free water. bound water and hydroxyl. and alkyl (methyl. methylene. and methine together) protons. A chemical shift and a line width were taken from a center of gravity and a full width at half height of the sum peak of the center peaks consisting of each group. However. accompanying spinuing side bands were included for peak area measurements.

Here bound water is defined as water exchanging fast with
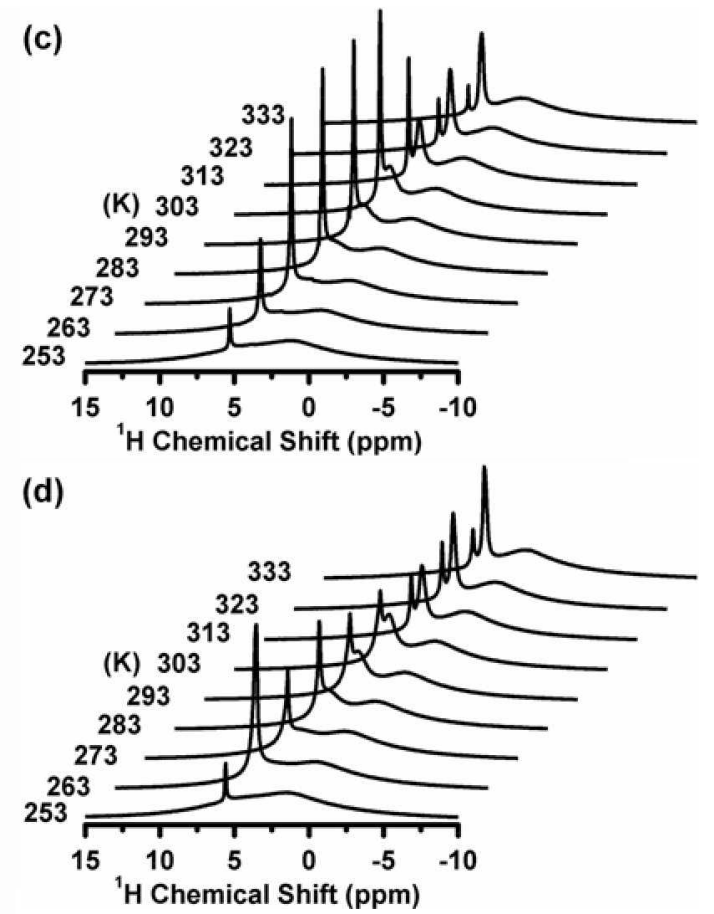

Figure 2. Stacked plot of ' $\mathrm{H}$ MAS spectra spun at $10 \mathrm{kHz}$ of (a) $9 \%$ PVB and (b) $18 \%$ PVB at room temperature and (c) $9 \%$ PVB and (d) $18 \%$ PVB at various temperatures. 


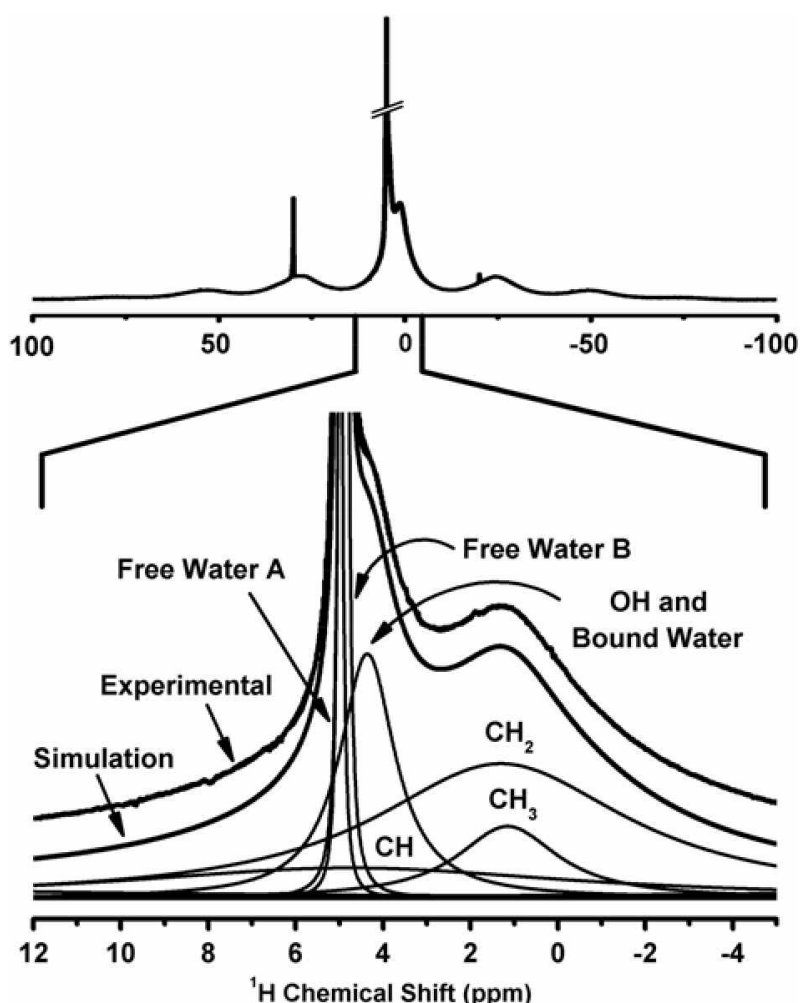

Figure 3. ${ }^{\text {H }}$ MAS spectrum of $18 \%$ PVB acquired at room temperature and $10 \mathrm{kHz}$ spinning rate. Full experimental spectrum (top), expanded center peak region and deconvoluted peaks (bottom). 'lhe experimental spectrum in expanded region was shifted upward from the sum spectrum of individual deconvoluted peaks for clarity.

Table 1. Peak Assignments of 'H MAS NMR Spectrum of $18 \%$ PVB at Room Iemperature

\begin{tabular}{ccc}
\hline \multicolumn{2}{c}{ Peak } & Assignment \\
\hline $\begin{array}{c}\text { Chemical Shift } \\
(\mathrm{ppm})\end{array}$ & $\begin{array}{c}\text { Full Width at Half } \\
\text { Height }(\mathrm{Hz})\end{array}$ & \\
\hline 5.0 & 54 & Free water proton $\mathrm{A}^{*}$ \\
4.9 & 18 & Free water proton $\mathrm{B}^{-}$ \\
4.7 & $5.0 \times 10^{3}$ & Methine protont \\
4.4 & $0.61 \times 10^{3}$ & Hydroxyl and bound \\
& & water proton \\
1.3 & $2.8 \times 10^{3}$ & Methylene proton \\
1.1 & $1.1 \times 10^{3}$ & Methyl proton \\
\hline
\end{tabular}

Free water peaks were deconwoluted into component $\mathrm{A}$ and $\mathrm{B}$. due to inherent nanoscale inhomogeneity. ${ }^{2}$ However, in the text and figures the two components are treated as a single peak representative of tree water.

hydrosyl protons resulting in a single peak for the bound water and hydroxyl protons. On the other hand. free water is far from hydroxyl groups. which hinders proton exchange between free water and hydroxyl groups as described in Figure 4

The proton quantities. converted from ${ }^{~} \mathrm{H}$ NMR peak areas, plotted as a function of spinning duration (Figure 5), indicate that the initial diminishing rate of free water was higher than that of bound water but at longer spinning times the rates of free and bound water depletion were similar.

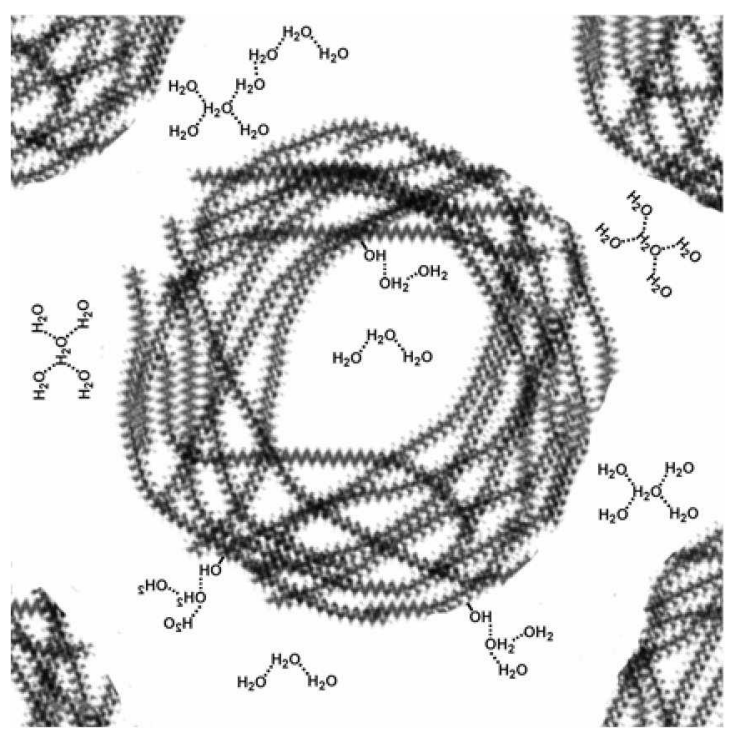

Figure 4. Schematic drawing of water in PVB: Dotted lines between water molecules indicate relatively tast proton exchange. Water exchanging protons with hydroxyl groups of PVB was defined as bound water, whereas water away from the bound water as free water. for more information on water in various states, refer to reterences $1-6$.

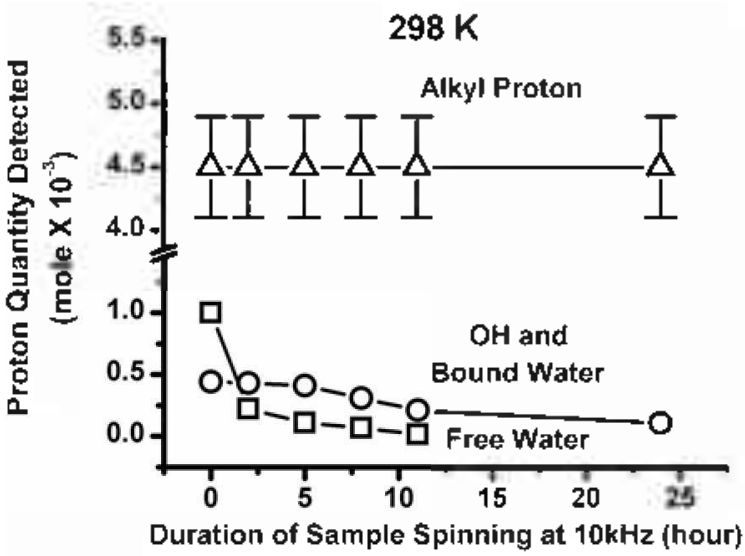

Figure 5. Proton quantity detected, calculated from the corresponding ${ }^{1} \mathrm{H}$ NMR peak areas, from tree water ( $\sqsupset$ ), bound water and hydroxyl ( $\because$ ) and alkyl proton ( -$)$ in $18 \%$ PVB spun at $10 \mathrm{kHz}$ versus spinning duration. All of the free water proton peaks were summed as a single peak. Likewise all alkyl proton proton (i.e' methine, methyl, and methyl protons) peaks were summed as a single alkyl proton peak. Reter to the main text for the proton quantity calculation procedure in detail. Solid lines are provided to guide the eyes.

However. the line width and chemical shift variation of all peaks was negligible (Figure 6). But for the chemical shift variation, it is also possible to assign some meaning to the small variation observed as follows. The free water peak shifted upfield marginally while the bound water and hy'droxyl peak shifted upfield more and then stayed at the same resonance frequency after the free water disappeared (Figure $6($ b)). At the same time the alkyl peak shifted downfield. These observations taken together imply the chemical shift of the bound water and hydroxyl protons can be an indicator of how much these protons interact with free water or PVB. 

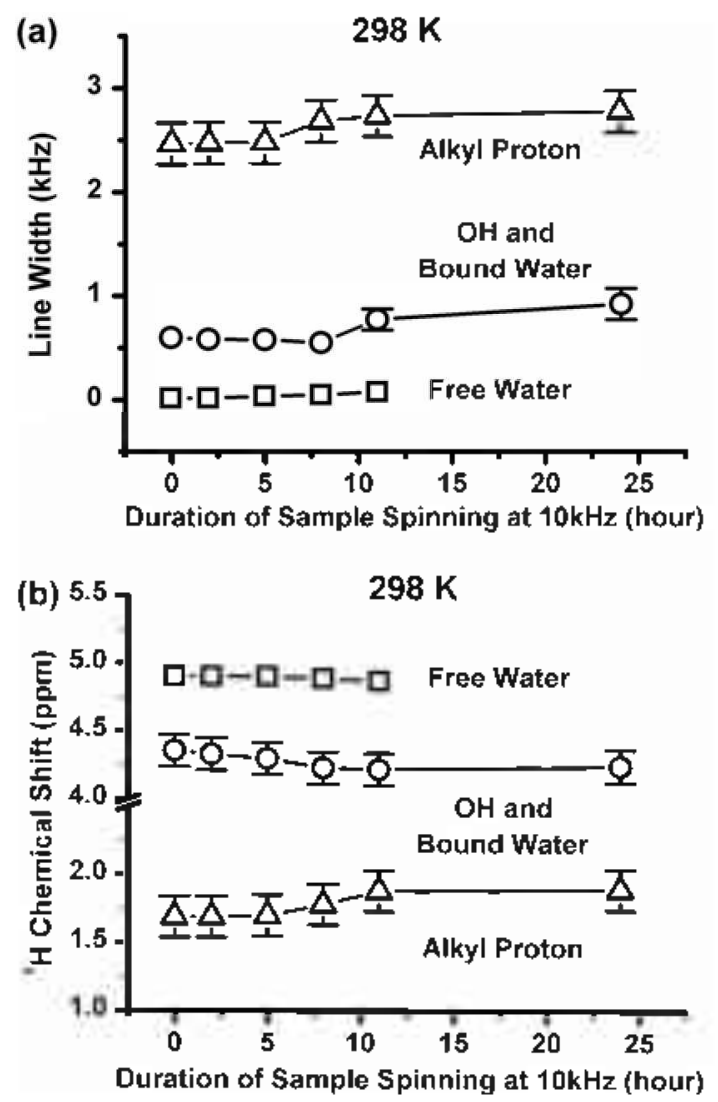

Figure 6. (a) line width and (b) chemical shift variation of free water ( $\sqsubset$ ), bound water and hydroxyl $(a)$, and alkyl $(-$ ) proton peaks of $18 \%$ PVB spun at $10 \mathrm{kHz}$ versus spimning duration. Solid lines are provided to guide the eves.

Proton MAS spectra acquired at various temperatures are shown in Figures 2(c) and 2(d). Initially the sample temperature was lowered to and kept at $253 \mathrm{~K}$ for a period longer than 2 hours to allow the sample to reach the temperature equilibrium before any NMR signal acquisition. Lower temperature experiments were done first to minimize water loss occurring more easily at higher temperature. Presence of the sharp free water peak even below $273 \mathrm{~K}$ clearly indicates the presence of some non-freezable free water. ${ }^{j 3}$ Proton peak of ice is too wide to be detected in this ${ }^{\mathrm{H}} \mathrm{H}$ MAS spectrum. The non-freezable free water must be located in nano pores ${ }^{1 \hat{j}}$ or near hydrophobic surface ${ }^{\hat{3}}$ Typically free water is regarded as free $\angle$ able and bound water as non-free $z$ able. ${ }^{1.3}$ Anisotropic ordering due to adjacent solid surfaces and reduced mobility make bound water non-freezable., ${ }^{1,3}$ Our observation shows some of our free water is non-freezable like non-freezable bound water in other literatures. ${ }^{1,3}$ This is not surprising since our definition of bound water was limited to water exchanging proton with hydroxyl groups of PVB

As the temperature was raised (under continuous spinuing at $10 \mathrm{kHz}$ ), the sharp free water peak started to grow from near $263 \mathrm{~K}$ due to melting. Above $303 \mathrm{~K}$ the free water peak intensity decreased: water was more easily expelled by evaporation due to higher temperature as well as by centrifugation from accumulated spiming time. As a result the

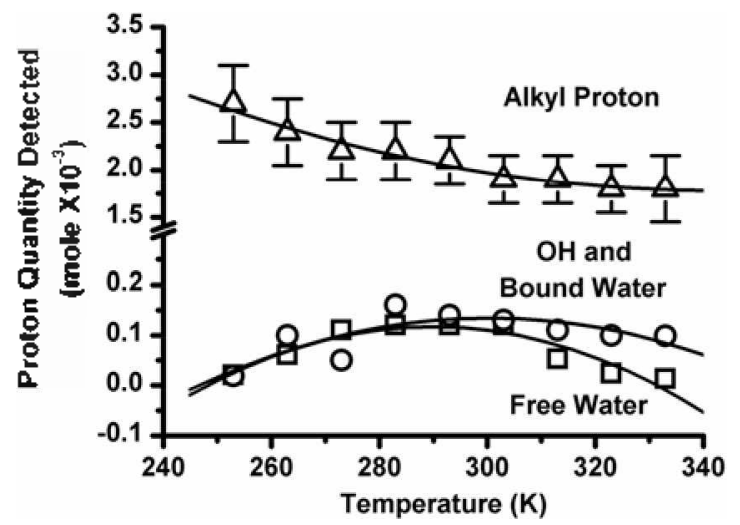

Figure 7. Proton quantity detected from free water $(\sqsupset)$, bound water and hydroxyl (o), and alkyl proton ( - ) in $9 \%$ PVB spun at $10 \mathrm{kHz}$ versus temperature. The proton quantities were converted from the corresponding peak areas relative to the spin counting data at room temperature. Refer to the main text for the conversion procedure in detail. Solid lines are provided to gujde the eyes.

water signal detected by the NMR coil was diminished. Greater information was provided by spectrum deconvolution. The peak areas of free water, bound water and hydroxyl group. and alkyl protons were converted to the proton quantities detected and plotted as a function of temperature in Figure 7, relative to the proton quantity measured at room temperature as described in the experimental section. Continuous depletion of the alkyl proton quantity detected at higher temperature mainly reflects NMR signal reduction from temperature dependence of Boltzmann distribution of proton spins. ${ }^{\text {Is }}$ In MAS spectra. sample spimning rates similar to the rate of molecular dynamics can induce peak broadening and accompanying signal reduction. ${ }^{15}$ However. this effect did not seem to play a major role for the reduction of the alkyl proton quantity detected. In fact. our line widths in Figure 8(a) slim down at higher temperature, manifesting the molecular dynamics at higher temperature deviated farther from our sample spinuing rate of $10 \mathrm{kHz}$. Thermal noise in NMR coils reduces detection limit and a signal-to-noise ratio at higher temperature. ${ }^{15}$ but its influence was negligible for our samples with big signals. The plot in Figure 7 reveals that bound water and hydroxyl protons have a similar behavior to free water: this suggests intimate interaction between free and bound water. There is no reason for hydroxyl group content to change unless condensation between hydroxyl groups occurs: therefore the intensity variation of the bound water and hydroxyl peak should mainly represent the variation of the bound water content. The depletion of proton detected from either bound or free water at higher temperature surpassed the anount reduced by temperature dependence of Boltzmann distribution. confinming that water was expelled by evaporation due to higher temperature as well as by centrifugation from accumulated spinning time.

The line width and chemical shift variation of ${ }^{1} \mathrm{H}$ MAS spectra of $9 \%$ PVB spun at $10 \mathrm{kH} /$ were plotted versus temperature in Figure 8(a) and (b), respectively. The line width of the bound water and hydroxyl peak is narrowed monotonically at higher temperature: greater molecular 

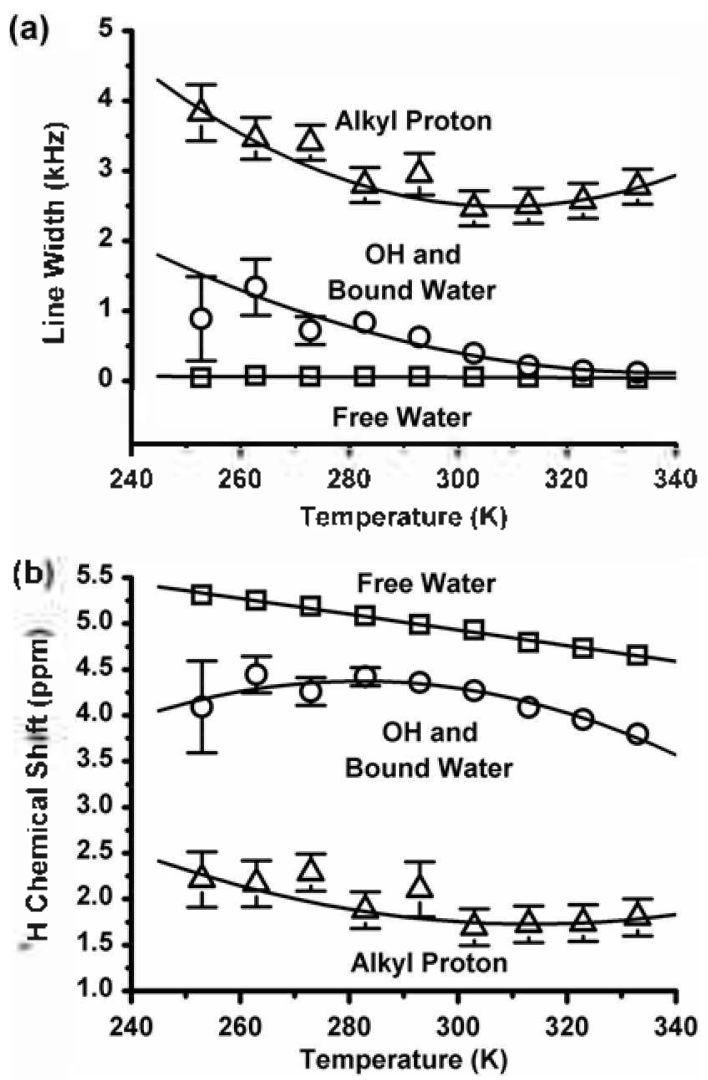

Figure 8. (a) line width and (b) chemical shift variation of ${ }^{-1} \mathrm{H}$ MAS spectra of $9 \%$ PVB spun at $10 \mathrm{kHz}$ versus temperature. The tree water, the bound water and hydroxyl, and the alkyl protons are denoted as $\sqsubset, \sigma$, and - respectively. Solid lines are provided to guide the eves.

motion at higher temperature causes greater averaging. The line width for free water is constant across temperature range. indicating that additional averaging does not narrow the line width further. In contrast. the alkyl peak line width decreases with increasing temperature and has a minimum near $300 \mathrm{~K}$ for both 18 and $9 \%$ PVB (Figure 8a). Previously' we reported that the minimum ${ }^{13} \mathrm{C}$ NMR line widths of PVB samples occurred at lower temperatures for higher contents of water. ${ }^{15}$ The similar behaviors of the line widths versus temperature in the region around $300 \mathrm{~K}$ indicate that similar water contents remained in the PVB and that the polymer molecular dynamics in both samples were similar. The chemical shift of the free water peak shifted upfield constantly as the temperature was raised while the bound water and hydroxyl peak and the alkyl peak had a maximum and minimum point near 285 and $305 \mathrm{~K}$. respectively in Figure 8 b. As the experiments progressed. the water depletion advanced due to accumulated spinning time and higher temperature. In turn, this water reduction induces upfield shifts of the free water and the bound water and hydroxyl peaks as observed in the room temperature data. At the same time as temperature is raised hydrogen bonding of water breaks more frequently ${ }^{1 \hat{3}}$ resulting in additional upfield shifts of the peaks. Lower temperature extends the bound water's residence time on PVB leading to upfield shifts. These two opposite shifting factors versus temperature bring about a maximum chemical shift near $285 \mathrm{~K}$. On the other hand. the minimum chenical shift of the alkyl protons near $305 \mathrm{~K}$ is due to the interaction with the bound waters causing a shift in the opposite direction to that of the bound water and hydroxyl peak as shown with the room temperature data.

\section{Conclusion}

${ }^{1} \mathrm{H}$ MAS NMR carried out at $10 \mathrm{kHz}$ in less than about 5 minutes was demonstrated to be a convenient and sensitive technique to measure: (a) the content variations of different types of water in polymers. (b) the degree of the interaction of water and polymer. and (c) the molecular dynamics of the polymer. The technique can be easily adapted to study different soft polymers with other small molecules than water in them. In our samples. polymer dy'namics was independent of the initial water content as long as the water contents of the samples at the measurement temperature were the same. possibly due to slow drying rates in our experiment. Drying rate dependence of polymer properties and difference of drying process by heat and by centrifugal force due to spinning will be studied in the near future.

Acknowledgement. This work was supported by the Grant R04-2002-000-00140-0 from KOSEF. Dr. Jong-Soo $\mathrm{Kim}$ at $\mathrm{OCI}$ and Sunha Kin at KBSI are thanked for generous supply of the PVB samples and teclunical assistance in NMR, respectively. In addition. Dr. S. H. Kin at KBSI is acknowledged for GC-MS experiments.

\section{References}

1. McBrierty. V. T.: Martit1. S. T.: Karasz. F. E. J. Mol Liqnids 1999. 80. 179-205

2. Mattos. C. TRENDS in Biochentical Sciences 2002. 27. 203-208.

3. van Oss. C. J.: Giese. R. F. J. Disp. Sci. Tech. 2004. 25. 631-655.

4. Bhattacharyya. S. M.: Wang. Z-G.: Zewail. A. H. J. Phns. Chent $B \mathbf{2 0 0 3}, 107.13218-13228$.

5. Geoghegana. M.: Krausch. G. Prog. Pohm. Sci. 2003. 28. 261-302.

6. Wiggins. P. M. Prog. Polym. Sci. 1988, 13, 1-35.

7. Cho. G.: Wu. Y.: Ackerman. T. L. Science 2003. 300. $1123-1127$.

8. Nagle. T. F.: Liu. Y.: Tristram-Nagle. S.: Epand. R. M.: Stark. R. E. Biophs. J. 1999. 77. 2062-2065.

9. Shin. J. E.: Comillon, P.: Salim. L. Food Hydrocolloids 2002. 16. 89-94.

10. Blomstrom. T. P. In Encyclopedia of Polyner Science and Engineering. 2nd ed.: Mark. H. F. Bikales. N.. Overberger. C. G.. Menges. G. Kroschwitz. J. I. Eds.: Wiley-Interscience: New York. 1989: Vol. 17. p 136.

11. Bruch, M. D.; Bonesteel. J.-A. K. Macromolecules 1986, 19. 1622-1627.

12. Schaefer J: McKay. R. A.: Stejskal. E. O.J. Hagi. Reson. 1983. 52. $123-129$

13. Haraficzyk. H.: Grandjean. J.: Olech. M. Colloids Surf. B 2003. 28. $239-249$

14. Hansen. E. W.: Schmidt. R.: Stocker, M. J. Phns. Chem. 1996. 100. 11396-114011.

15. Pfeifer, H. In Mi R Basic Principles and Progess, Solid-State MM $R$ II: Inorganic Hatter. Diehl. P.. Fluck. E.. Gutther. H. Kosteld. R. Seelig. J.. Eds.: Springer-Verlag: Berlin, 1994: Vol. 31. pp 31-90.

16. Namgoong. H.: Kim. T. S.: Han. O. H. J. Kor. Magn. Reson. Soc. 2002. $6,1-11$ 